\title{
Upregulation of exosomal microRNA-21 in pancreatic stellate cells promotes pancreatic cancer cell migration and enhances Ras/ERK pathway activity
}

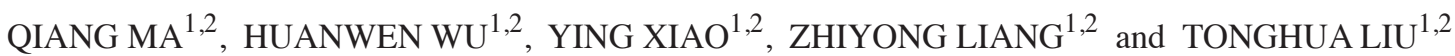 \\ ${ }^{1}$ Department of Pathology, Peking Union Medical College Hospital; ${ }^{2}$ Center for Molecular Pathology, \\ Chinese Academy of Medical Sciences and Peking Union Medical College, Beijing 100730, P. R. China
}

Received August 19, 2019; Accepted January 20, 2020

DOI: 10.3892/ijo.2020.4986

\begin{abstract}
Pancreatic stellate cells (PSCs) are typically activated in pancreatic ductal adenocarcinoma (PDAC) and release exosomes containing high levels of microRNA-21 (miR-21). However, the specific roles of exosomal miR-21 in regulating the PDAC malignant phenotype remain unknown. The present study aimed to determine the effects of exosomal miR-21 on the migratory ability of PDAC cells and explore the potential underlying molecular mechanism. Weighted gene correlation network and The Cancer Genome Atlas database analysis revealed that high miR-21 levels were associated with a poor prognosis in patients with pancreatic adenocarcinoma, and that the Ras/ERK signaling pathway may be a potential target of miR-21. In vitro, PDAC cells were demonstrated to internalize the PSC-derived exosome, resulting in high miR-21 levels, which subsequently promoted cell migration, induced epithelial-to-mesenchymal transition (EMT) and increased matrix metalloproteinase-2/9 activity. In addition, exosomal miR-21 increased the levels of ERK1/2 and Akt phosphorylation in PDAC cells. Collectively, these results suggested that PSC-derived exosomal miR-21 may promote PDAC cell migration and EMT and enhance Ras/ERK signaling activity. Thus, miR-21 may be a potential cause of poor prognosis in patients with pancreatic cancer and a new treatment target.
\end{abstract}

\section{Introduction}

Pancreatic ductal adenocarcinoma (PDAC) is the most common form of pancreatic cancer (1), with an estimated 458,918 diagnoses and 432,242 deaths from pancreatic cancer globally in 2018 (2), mainly due to its high degree of inva-

Correspondence to: Dr Zhiyong Liang, Department of Pathology, Peking Union Medical College Hospital, 1 Wangfujing Street, Beijing 100730, P.R. China

E-mail: liangzhiyong1220@yahoo.com

Key words: pancreatic ductal adenocarcinoma, pancreatic stellate cells, exosomal miR-21, migration siveness and metastatic potential $(1,3)$. Therefore, continued research of the molecular mechanisms underlying PDAC metastasis are essential for identifying new targets to prevent tumor progression and recurrence.

Exosome-mediated intercellular communication is an important factor in regulating malignant cell behavior (4). In particular, exosomal microRNAs (miRNAs) induce tumor progression by downregulating the expression of tumor suppressor genes $(5,6)$. Among the known exosomal miRNAs, miRNA (miR)-21 is a well-established oncogenic miRNA that serves various cancer-specific roles, including those associated with metastasis. For example, exosomes derived from hypoxic oral squamous cell carcinoma cells deliver miR-21 to normoxic cells to induce a pro-metastatic phenotype (7); in addition, exosomal miR-21 has been demonstrated to promote the migration and invasion of esophageal cancer cells by targeting programmed cell death-4 (8). The expression level of miR-21 has been reported to be elevated in pancreatic stellate cell (PSC)-derived exosomes in patients with PDAC (9-11). PSCs are a special type of cancer-associated fibroblasts that are often abnormally activated in PDAC and are closely associated with PDAC progression; however, to the best of our knowledge, the specific functions of miR-21 and its underlying molecular mechanisms in regulating PDAC cell malignancy have not been elucidated (12-19).

The present study investigated the differences in miR-21 expression profiles in PDAC and normal tissues based on public databases, and explored effect and potential mechanism of PSC-derived exosomal miR-21 on the development of PDAC-related malignant phenotypes.

\section{Materials and methods}

Datasets and analysis. Data on miRNA expression in pancreatic adenocarcinoma (PAAD) tissue were downloaded from The Cancer Genome Atlas (TCGA) (http://cancergenome.nih. gov/) (20). Clinical data, survival time and KRAS mutation status of patients with PAAD were also downloaded from the cBioPortal database (www.cbioportal.org) (21). Weighted gene correlation network analysis (WGCNA) was used for miRNA data analysis, and GS was calculated by $\mathrm{R}$ software with the WGCNA package (15). Kyoto Encyclopedia of Genes and 
Genomes (KEGG) pathway enrichment analysis of miR-21 target genes was conducted using the Search Tool for the Retrieval of Interacting Genes database (https://string-db. org) (22). The effects of the KRAS mRNA expression levels on the survival of patients with PAAD were further evaluated using the UALCAN database (http://ualcan.path.uab. edu/index.html) (14).

Cell culture and transfection. Human PDAC cell lines PANC-1 and MIAPaCa-2 were obtained from the American Type Culture Collection and maintained in DMEM (Corning, Inc.) supplemented with 10\% FBS (Corning, Inc.) and $1 \%$ penicillin and streptomycin $(100 \mathrm{U} / \mathrm{ml}$; Cell Resource Center of Peking Union Medical College). Human PSCs were isolated from three fresh surgically resected pancreatic cancer tissues (three male patients; mean age, 60.67 years; age range, 56-64 years; diagnosed as PDAC by pathological examination between January and August 2017), which were cut into $\sim 1-\mathrm{mm} 3$ sections using sterile tweezers and scissors, followed by washing twice with PBS (Corning, Inc.) containing $1 \%$ penicillin and streptomycin. The tissue blocks were seeded in 6-well culture plates in the presence of $1 \mathrm{ml}$ DMEM/F-12 (1:1; Corning, Inc.) containing 10\% FBS and $1 \%$ penicillin and streptomycin (12), and the culture medium was changed every $24 \mathrm{~h}$. The cells were cultured at $37^{\circ} \mathrm{C}$ in an atmosphere with $5 \% \mathrm{CO}_{2}$.

For small interfering RNA (siRNA) transfection, the PSCs were transfected with an miR-21 inhibitor or negative-control (NC) oligonucleotides (Table I; Shanghai GenePharma Co., Ltd.) using Lipofectamine ${ }^{\circledast} 2000$ (Thermo Fisher Scientific, Inc.) according to the manufacturer's instructions. The cells were cultured for $24 \mathrm{~h}$ at $37^{\circ} \mathrm{C}$ in an atmosphere with $5 \% \mathrm{CO}_{2}$.

Isolation and identification of exosomes. After reaching 90\% confluence, PSCs and transfected PSCs were washed with PBS and incubated in freshly prepared DMEM/F12 without FBS for $48 \mathrm{~h}$ at $37^{\circ} \mathrm{C}$. Exosomes were isolated from the conditioned medium by differential centrifugation (Optima L-100XP ultracentrifuge; Beckman Coulter, Inc.) as previously described (13). Following ultracentrifugation, the isolated exosomes were dropped to the copper mesh, precipitated for $2 \mathrm{~min}$, and filter paper was used to absorb the excess sample. After drying the copper mesh on the filter paper for $5 \mathrm{~min}, 1 \%$ uranyl acetate was added for $2 \mathrm{~min}$ and dried for 40 min. Transmission electron microscopy (TEM; EM-1400 plus; JEOL, Ltd.) was used to assess the morphological characteristics of exosomes (magnification, $\mathrm{x} 80,000$ ). The PSCs were fixed with $2.5 \%$ glutaraldehyde at $4^{\circ} \mathrm{C}$ for $2 \mathrm{~h}$; electron microscopy was used to observe ultrastructural changes (magnification, $x 50,000)$. The exosome concentration was determined using a bicinchoninic acid assay (Thermo Fisher Scientific, Inc.) according to the manufacturer's instructions.

Exosome labeling. Exosomes isolated from PSCs were suspended in $100 \mu \mathrm{l}$ PBS, followed by the addition of $1 \mathrm{ml}$ Diluent $\mathrm{C}$ from the PKH67 Fluorescent Cell Linker kit (Sigma-Aldrich; Merck KGaA). PKH67 buffer $(2 \mu \mathrm{l})$ was then added to the solution, and following a 3-min incubation at $25^{\circ} \mathrm{C}, 4 \mathrm{ml}$ serum-free DMEM/F12 was added to terminate the labeling reaction. After an additional $1 \mathrm{~min}$, the medium was added to a sub-confluent layer of PANC-1 or MIAPaCa-2 cells, which were then incubated at $37^{\circ} \mathrm{C}$ for $3 \mathrm{~h}$. The cells were washed twice with PBS and observed under an IX71 fluorescence microscope (Olympus Corporation; magnification, $\mathrm{x} 400)$.

Migration assays. For wound-healing assays, PANC-1 or MIAPaCa-2 cells were seeded into 6-well plates ( $5 \times 10^{5}$ cells/well). Upon reaching $\sim 90 \%$ confluence, the monolayer was scratched using a $200-\mu 1$ pipette tip, followed by culture in $2 \mathrm{ml}$ DMEM without FBS. The relative scratch width was determined at 0,24 and $48 \mathrm{~h}$ using an inverted light microscope (Olympus Corporation). ImagePro Plus software (v.6.0; Media Cybernetics, Inc.) was used to calculate the average relative scratch width as follows: Relative scratch width $=$ wound area $/$ wound height.

Transwell assays were performed to validate the results of the wound-healing assays. PANC- 1 and MIAPaCa- 2 cells suspended in $100 \mu 1$ DMEM containing $0.05 \%$ FBS were added to the upper chambers of Transwell plates $\left(1 \times 10^{5}\right.$ cells/well; Corning, Inc.), and $600 \mu \mathrm{l}$ DMEM with $10 \%$ FBS was added to the lower chambers of each well. After incubation at $37^{\circ} \mathrm{C}$ for $16 \mathrm{~h}$, the chambers were fixed with $4 \%$ PFA for $15 \mathrm{~min}$ at $25^{\circ} \mathrm{C}$, stained with $0.5 \%$ crystal violet for $10 \mathrm{~min}$ at $25^{\circ} \mathrm{C}$ and washed twice with PBS. Migrated cells were counted in five randomly selected fields under an inverted light microscope (Olympus Corporation).

Reverse transcription-quantitative PCR (RT-qPCR). Total RNA was extracted from PSCs (untreated, and transfected PSCs), PANC-1 and MIAPaCa- 2 cells (treated and untreated with PSCs) and their exosomes using TRIzol $^{\circledR}$ reagent (Invitrogen; Thermo Fisher Scientific, Inc.) according to the manufacturer's instructions. miR-21 was reverse-transcribed to cDNA using a High-Capacity cDNA Reverse Transcription Kit (Applied Biosystems; Thermo Fisher Scientific, Inc.) using a miRNA-specific stem-loop reverse transcriptase primer $\left(25^{\circ} \mathrm{C}\right.$ for $1 \mathrm{~min}, 37^{\circ} \mathrm{C}$ for $120 \mathrm{~min}$ and $85^{\circ} \mathrm{C}$ for $5 \mathrm{~min}$ ); the first-strand cDNA was used as a template for qPCR. PCRs were run using a Maxima $\mathrm{SYBR}^{\circledR}$ Green qPCR master mix (Applied Biosystems, Foster City, CA, USA) on an ABI 7500 real-time PCR detection system (Applied Biosystems; Thermo Fisher Scientific, Inc.) using the following thermocycling conditions: $95^{\circ} \mathrm{C}$ for $60 \mathrm{sec}$, followed by 40 cycles of $95^{\circ} \mathrm{C}$ for $10 \mathrm{sec}, 60^{\circ} \mathrm{C}$ for $30 \mathrm{sec}$ and $72^{\circ} \mathrm{C}$ for $10 \mathrm{sec}$. All reactions were run in triplicate, and the $2^{-\Delta \Delta \mathrm{Ct}}$ method (23) was used to determine relative gene expression levels using U6 as an internal control (Table I).

Western blotting. Different amounts of PSC-derived exosomes $(0,5,10$ or $20 \mu \mathrm{g})$ were added to PANC- 1 cells. After $48 \mathrm{~h}$, the cells and exosomes were collected and lysed in radioimmunoprecipitation assay buffer (Applygen Technologies, Inc.) in the presence of protease and phosphatase inhibitors (Thermo Fisher Scientific, Inc.) for $30 \mathrm{~min}$ to extract total proteins, which were quantified by a bicinchoninic acid assay (Thermo Fisher Scientific, Inc.). Proteins ( $40 \mu \mathrm{g} / \mathrm{lane})$ were separated by $10 \%$ SDS-PAGE and transferred to polyvinylidene difluoride membranes (EMD Millipore), which were blocked with 5\% skimmed milk powder (BD Biosciences) for $1 \mathrm{~h}$ at 
Table I. Primers and inhibitors used in the present study.

\begin{tabular}{lll}
\hline Name & \multicolumn{1}{c}{ Type } & \multicolumn{1}{c}{ Sequence $\left(5^{\prime} \rightarrow 3^{\prime}\right)$} \\
\hline miR-21 & Stem-loop & GTTGGCTCTGGTGCAGGGTCCGAGGTATTCGCACCAGAGCCAACTCAACA \\
& Forward & GCGGCGTAGCTTATCAGACTGA \\
& Reverse & GTGCAGGGTCCGAGGT \\
U6 & Forward & CTCGCTTCGGCAGCACA \\
NC inhibitor & Reverse & AACGCTTCACGAATTTGCGT \\
miR-21 inhibitor & Sense & CAGUACUUUUGUGUAGUACAA \\
\end{tabular}

miR, microRNA; NC, negative control.

$25^{\circ} \mathrm{C}$. The membranes were subsequently incubated at $4^{\circ} \mathrm{C}$ overnight with the following primary antibodies: CD63 (1:30; cat. no. D360973), CD9 (1:30; cat. no. D264336; both from Sangon Biotech Co., Ltd.), ERK1/2 (1:1,000; cat. no. 9102S), phospho-ERK1/2 (Thr202/Tyr204; 1:1,000; cat. no. 9101S), Akt (1:1,000; cat. no. 4691), phospho-Akt (Ser473; 1:1,000; cat. no. 4060P), vimentin (1:1,000; cat. no. 5741T), N-cadherin (1:1,000; cat. no. 13116), $\beta$-catenin (1:1,000; cat. no. 8480), Snail $(1: 1,000$; cat. no. 3879T) and E-cadherin (1:1000; cat. no. 3195T; all from Cell Signaling Technology, Inc.). The membranes were subsequently incubated with horseradish peroxidase (HRP)-conjugated goat anti-rabbit/mouse secondary antibodies (cat. nos. ZB-5301 and ZB-5305; 1:10,000; Beijing Zhongshan Golden Bridge Biotechnology Co., Ltd.) at room temperature for $1 \mathrm{~h}$. Immunocomplexes were detected using Immobilon western chemiluminescent HRP substrate (EMD Millipore), and images were captured using the ChemiDoc MP imaging system with Image Lab Touch software (v.2.0; Bio-Rad Laboratories, Inc.).

Gelatin zymography. PANC-1 cells were seeded in 6-well plates at $5 \times 10^{5}$ cells/well and incubated in DMEM with $10 \% \mathrm{FBS}$ at $37^{\circ} \mathrm{C}$ for $24 \mathrm{~h}$. Subsequently, the medium was replaced with serum-free DMEM, and the exosomes from untreated PSCs or PSCs treated with the miR-21 inhibitor were added to the wells. After $24 \mathrm{~h}$, culture supernatants were collected, and $40 \mu 1$ of each supernatant per lane was loaded into a $10 \%$ SDS-PAGE gel containing $1 \mathrm{mg} / \mathrm{ml}$ gelatin (Sigma-Aldrich; Merck KGaA). Following electrophoresis, the gel was washed twice for 30 min with $2.5 \%$ Triton X-100 at room temperature and incubated in zymography buffer [50 mM Tris- $\mathrm{HCl}(\mathrm{pH} 7.5), 200 \mathrm{mM} \mathrm{NaCl}, 10 \mathrm{mM} \mathrm{CaCl}_{2}$ and $\left.1 \mu \mathrm{M} \mathrm{ZnCl}_{2}\right]$ at $37^{\circ} \mathrm{C}$ for $24 \mathrm{~h}$. The gel was subsequently stained for $2 \mathrm{~h}$ in $0.25 \%$ Coomassie Brilliant Blue R-250 and destained with destaining buffer (methanol: glacial acetic acid: distilled water, 3:1:6). A clear white band indicated the presence of matrix metalloproteinase-2 or -9 (MMP-2/9) activity. The gels were photographed using the ChemiDoc MP imaging system (Bio-Rad Laboratories, Inc.).

Statistical analysis. Data were analyzed using SPSS (v.21.0; IBM Corp.), R software (v.3.4.3; https://www.r-project. org/; 'WGCNA' package) and GraphPad Prism 5 (GraphPad
Software, Inc.). A log-rank test was used to analyze the Kaplan-Meier curve to evaluate the association between $K R A S$ expression and the survival rate. The data from the wound-healing and Transwell assays were analyzed by one-way analysis of variance followed by Tukey's post hoc test. $\mathrm{P}<0.05$ was considered to indicate a statistically significant difference.

\section{Results}

High miR-21 expression levels are associated with poor prognosis and Ras pathway activation in patients with pancreatic cancer. To investigate whether miR-21 serves a key role in pancreatic cancer pathogenesis and progression, the relationship between miRNA expression levels in tumor tissues and prognosis in patients with pancreatic cancer were analyzed using the WGCNA approach based on TCGA data. In the WGCNA, miR-21 was located in the turquoise module, which correlated well with overall survival (OS), disease-free survival (DFS) and tumor grade $(\mathrm{P}<0.05$; Fig. $1 \mathrm{~A}$ and B). Specifically, the gene significance value of miR-21 was positively correlated with tumor grade and negatively correlated with OS and DFS $(\mathrm{P}<0.05$; Fig. 1B). KEGG analysis revealed the primary involvement of miR-21 target genes in the Ras and ERK pathways (Fig. 1C). In addition, database analysis revealed that the survival probability of patients with high $K R A S$ expression was lower compared with that in patients with low/medium $K R A S$ expression ( $\mathrm{P}=0.023$; Fig. 1D). The frequency of KRAS mutations was $91 \%$ in patients with PAAD (Fig. 1E). These results suggested that miR-21 may be associated with the activation of the Ras pathway in pancreatic cancer.

PDAC cells internalize exosomal miR-21 from PSCs. TEM images of the ultrastructure of PSCs revealed numerous multivesicles in the cytosol containing varying amounts of vesicles (Fig. 2A and B). Membrane structures of 40-100 nm in size were observed in the extracellular vesicles (Fig. 2C, red arrows), which exhibited strong expression of the exosomal markers CD63 and CD9 (Fig. 2D). This result confirmed that the isolated exosomes were obtained from the conditioned media of PSC cultures. To determine whether PDAC internalized PSC-derived exosomes, PSC-derived exosomes were labeled with the dye PKH67 and added to MIAPaCa-2 
A

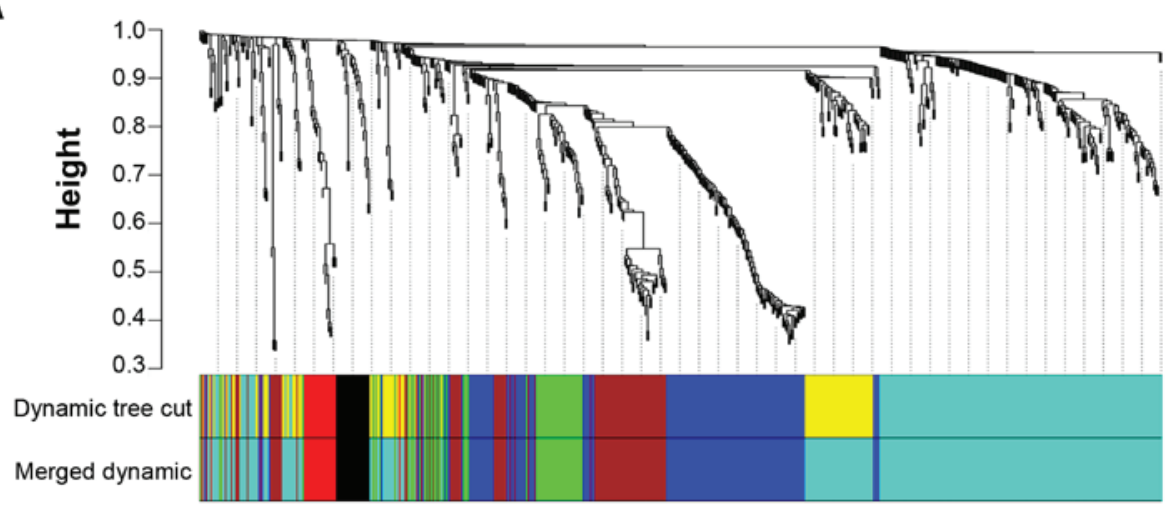

B

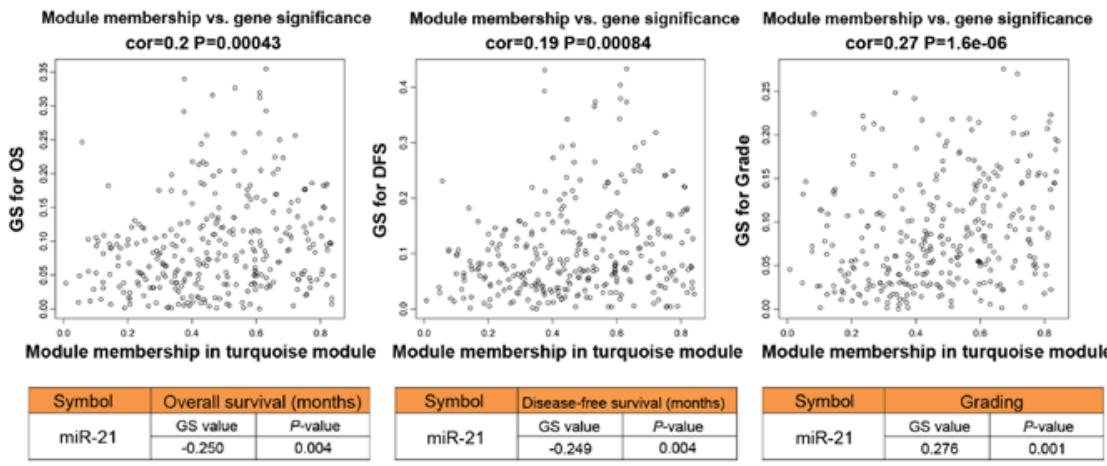

C

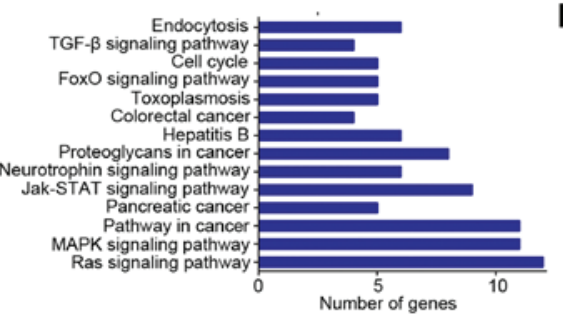

D Effect of KRAS expression level on PAAD patient survival

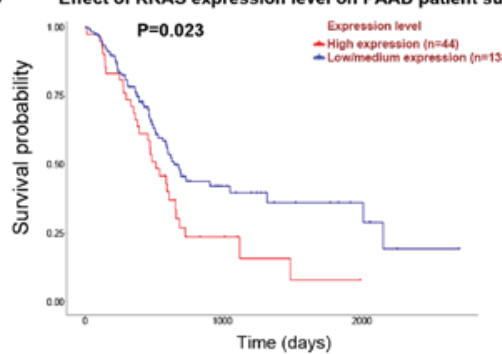

E

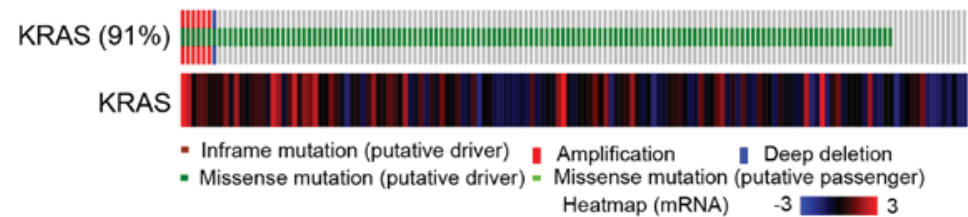

Figure 1. miR-21 level is a key factor in the prognosis of patients with PDAC and is associated with the Ras signaling pathway. (A) miRNA expression in patients with PAAD according to data from TCGA database $(\mathrm{n}=131)$ and WGCNA analysis. Samples were divided based on the co-expression of the miRNAs into several subsets by hierarchical clustering, with branches representing different gene modules (represented by a specific color) and miRNAs within the modules exhibiting high degrees of co-expression. (B) The GS of members of the turquoise module was analyzed by WGCNA, indicating significant correlations with OS, DFS and tumor grade in patients with PAAD. (C) The primary signaling pathways involving miR-21 target genes. (D) Effects of KRAS expression levels on PAAD patient survival time based on data from the UALCAN and TCGA databases (n=177). (E) $K R A S$-mutation status in patients with pancreatic cancer $(\mathrm{n}=149)$. miR, miRNA, microRNA; WGCNA, weighted gene correlation network analysis; PDAC, pancreatic ductal adenocarcinoma; PAAD, pancreatic adenocarcinoma; TCGA, The Cancer Genome Atlas; OS, overall survival; DFS, disease-free survival; GS, gene significance.

and PANC-1 cultures. After 3 h, fluorescent exosomes were detected inside these cells (Fig. 2E), indicating their effective uptake. In addition, miR-21 levels were increased in these cells compared with untreated cells $(\mathrm{P}<0.05$; Fig. $2 \mathrm{E})$.

PSC-derived exosomal miR-21 induces PDAC cell migration. The miR-21 inhibitor was transfected into PANC-1 and MIAPaCa-2 cells, and relative miR-21 levels were decreased compared with the negative control group $(\mathrm{P}<0.05$; Fig. $3 \mathrm{~A})$, which indicated that miR-21 was successfully transfected into the two cells lines. To determine whether the migra- tion-promoting effect of PSCs was mediated by exosomes, wound-healing and Transwell assays were performed. The results of the wound-healing assay demonstrated that MIAPaCa-2 and PANC-1 cells treated with exosomes for $48 \mathrm{~h}$ migrated over longer distances compared with the cells in the respective control groups $(\mathrm{P}<0.05$; Fig. 3B). Additionally, Transwell assays demonstrated a higher number of MIAPaCa-2 and PANC-1 cells migrating across the membrane following exosome treatment compared with those observed in the control groups $(\mathrm{P}<0.05$; Fig. 3C). In addition, the two assays revealed that the migratory ability of PDAC cells decreased 


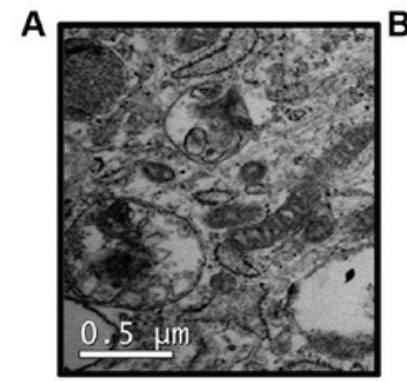

E
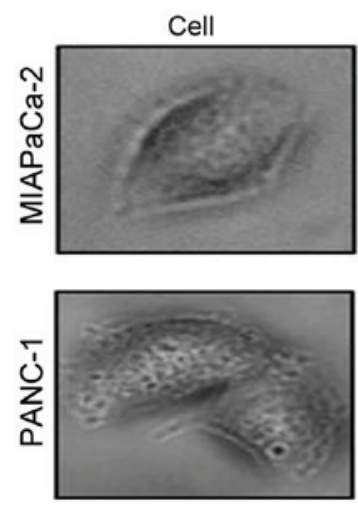

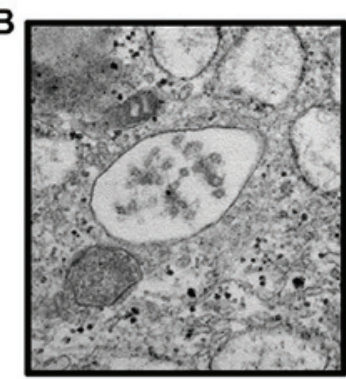

PKH67
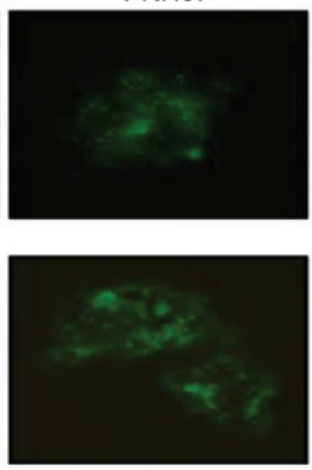
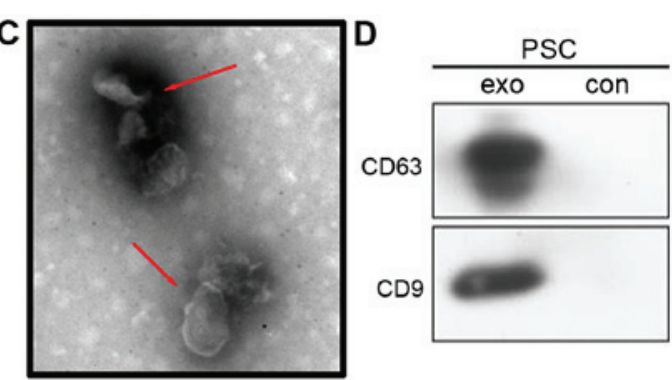

Merged
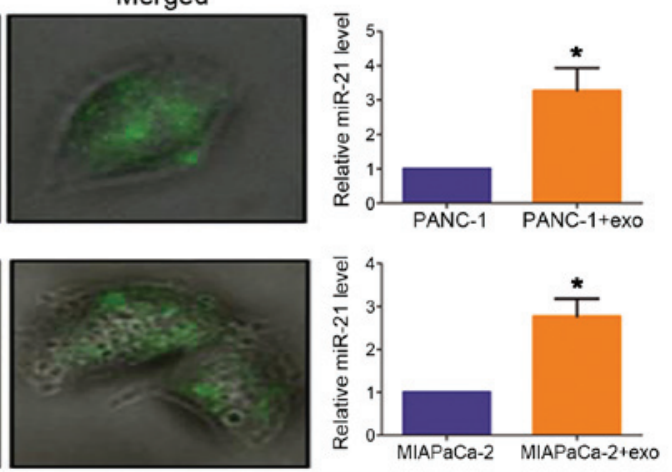

Figure 2. Activated PSC-derived exosomes exhibit high miR-21 levels and can be taken up by PDAC cells. (A and B) Representative TEM images of the PSC ultrastructure and multivesicles. (C) A representative TEM image of exosomes (red arrows) isolated from PSC-conditioned medium. (D) Western blots of the exosomal markers CD63 and CD9 in exosome-enriched conditioned medium and lysate. (E) PSC-derived exosomes labeled with PKH67 (green) were taken up by MIAPaCa-2 and PANC-1 cells (magnification, $\mathrm{x} 400$ ). Bar graphs present the results of the quantitative analysis of relative miR-21 levels. ${ }^{*}<0.05$, PANC-1+EXO vs. PANC1 or MIAPaCa-2+EXO vs. MIAPaCa-2. PSC, pancreatic stellate cell; miR, microRNA; TEM, transmission electron microscopy; exo, exosome-enriched conditioned medium; con, control.

significantly following treatment with exosomes from PSCs transfected with the miR-21 inhibitor compared with that observed in cells treated with exosomes derived from miR-NC-treated PSCs (Fig. 3B and C).

PSC-derived exosomal miR-21 induces epithelial-mesenchymal transition (EMT) and promotes MMP-2/9 expression in PDAC cells. The EMT process and MMP-2/9 activity are widely regarded as vital factors involved in tumor metastasis, and EMT and pancreatic tumor aggressiveness are reportedly strongly associated $(17,18)$. Therefore, it was hypothesized that PSC-derived exosomal miR-21 may induce EMT. To test this hypothesis, the levels of EMT biomarkers in MIAPaCa-2 and PANC-1 cells were determined following treatment with PSC-derived exosomes. As demonstrated in Fig. 4A and B, the levels of N-cadherin, Snail and vimentin were considerably increased, whereas E-cadherin expression was downregulated in the cells treated with PSC-derived exosomes. By contrast, inhibition of exosomal miR-21 decreased N-cadherin, Snail and vimentin expression levels and increased E-cadherin expression levels in MIAPaCa-2 and PANC-1 cells (Fig. 4A and B). In addition, MMP-2/9 activity increased in exosome-treated cells compared with untreated controls and cells treated with the PSC supernatant (NC) (Fig. 4C). Inhibition of PSC-derived exosomal miR-21 decreased MMP-2/9 activity in PANC-1 cells compared with that observed in cells treated with exosomes from miR-NC-treated PSCs (Fig. 4D).

Exosomal miR-21 enhances the Ras/ERK and Ras/Akt signaling pathway activity in PDAC cells. Activation of the
$\mathrm{K}-\mathrm{Ras} / \mathrm{Akt}$ and K-Ras/ERK signaling pathways induced by $K R A S$ alterations has been reported to be a primary cause of pancreatic cancer progression (24-27). Since the KEGG pathway analysis suggested that miR-21 was associated with Ras pathway activation, it was hypothesized that PSC-derived exosomal miR-21 may be involved in regulating the activation of the Ras/ERK and Ras/Akt signaling pathways. In support of this hypothesis, the addition of PSC-derived exosomes to the PANC-1 cell culture gradually increased the levels of Akt phosphorylation in proportion with the amount of exosomes added, with phosphorylation levels peaking at $20 \mu \mathrm{g}$ (Fig. 4E). The level of ERK phosphorylation also gradually increased along with increasing exosome number, although phosphorylation levels peaked at $10 \mu \mathrm{g}$ rather than $20 \mu \mathrm{g}$, which may indicate a dose-dependent effect within a certain range (Fig. 4E). To confirm that these signaling pathways were activated by exosomal miR-21, $10 \mu \mathrm{g}$ exosomes from PSCs treated or with the miR-21 inhibitor or miR-NC were added to PANC-1 and MIAPaCa- 2 cell cultures and incubated for $48 \mathrm{~h}$. Inhibition of exosomal miR-21 resulted in notable decreases in ERK and Akt phosphorylation levels in PANC-1 cells compared with those in the NC groups, although this change was not obvious in MIAPaCa-2 cells (Fig. $4 \mathrm{~F}$ and $\mathrm{G}$ ). These results suggested that exosomal miR-21 may induce the activation of the Ras/ERK and Ras/Akt signaling pathways.

\section{Discussion}

The results of the present study demonstrated that PSC-derived exosomal miR-21 was internalized by PDAC cells and 
A

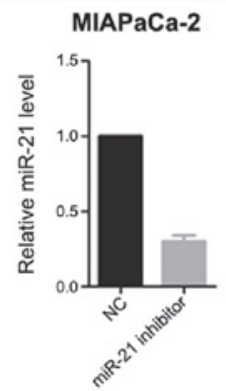

B

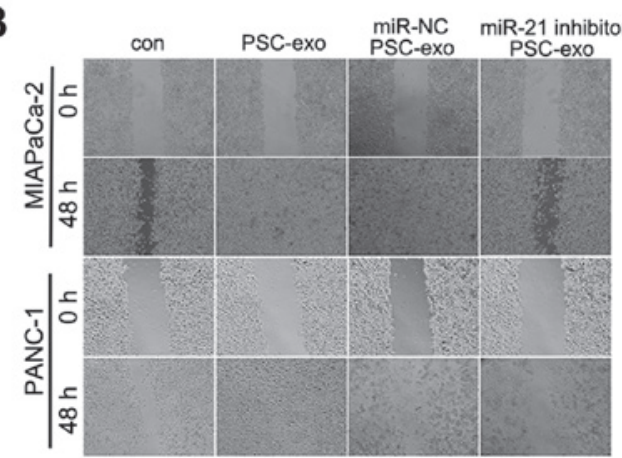

C

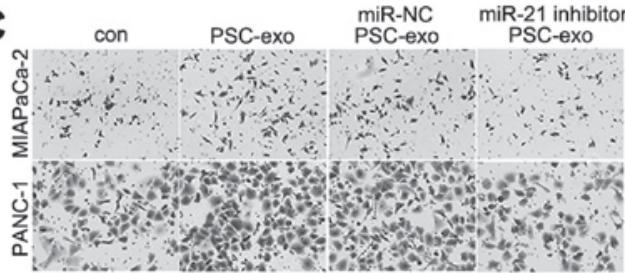

PANC-1

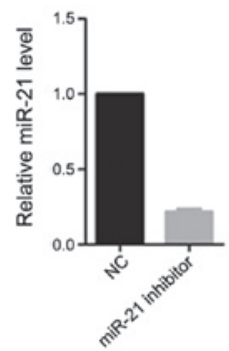

- con 21 inhibitor PSC-exo miR-NCPSC-exo
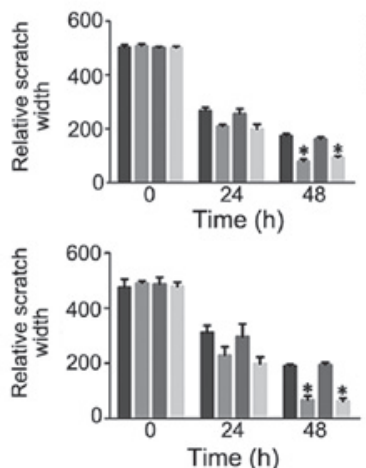

- ${ }_{\text {miR-21 inhibitor PSC-exo }}^{c o n}$ PSC-exo
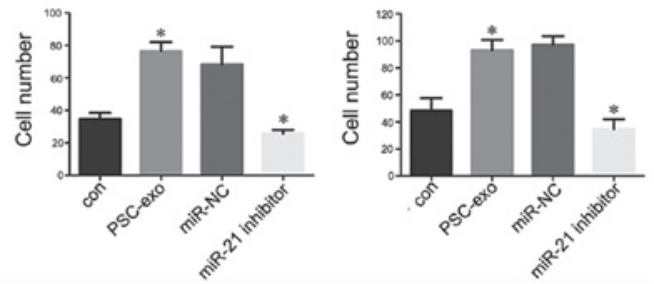

Figure 3. PSC-derived exosomal miR-21 promotes the migration of pancreatic cancer cells. (A) miR-21 levels were detected by qPCR in PANC-1 and MIAPaCa-2 cells following transfection with the miR-21 inhibitor and NC. (B) Wound-healing assay results at 0, 24 and 48 h. (C) Transwell assay results demonstrating the migratory ability of MIAPaCa-2 and PANC-1 cells. * P<0.05 PSC-exo vs. con or miR-21 inhibitor PSC-exo vs. miR-NC PSC-exo. miR, microRNA; qPCR, quantitative PCR; NC, negative control; PSC, pancreatic stellate cell; exo, exosome-enriched conditioned medium; con, control.

subsequently modulated the migratory capacity and motility of PDAC cells by enhancing the EMT process and increasing MMP-2/9 activity in addition to enhancing Ras/ERK and Ras/Akt signaling activation (Fig. 4H). These results suggested a novel role of exosomal miR-21 in the progression and invasiveness of pancreatic cancer.

Previous studies have reported that abnormally activated PSCs contribute to PDAC progression, including the induction of pancreatic fibrosis (28), collagen production (29) and cell-cell interactions (30). However, the underlying molecular mechanisms have not been completely elucidated. Recently, cancer-associated fibroblast-derived exosomes have attracted attention as regulators of tumor malignant phenotypes (31), which has been validated in ovarian (13), colorectal (32), breast (33) and other types of cancer. Therefore, the results of the present study demonstrating the effects of PSC-derived exosomes in promoting the EMT process and migratory capacity of PDAC cells are in accordance with previous observations. Additionally, these results may contribute further insight into the mechanism of PSC-induced tumor metastasis.

Exosomes mediate intercellular cross-talk under physiological and pathological conditions through the transfer of miRNAs (34). To explore the molecular mechanisms underlying the processes observed in the present study, exosome-associated miRNAs were analyzed. miR-21 is a well-known oncogenic miRNA that promotes tumor progression by inhibiting the expression of tumor-suppressor genes $(35,36)$ and is associated with high metastatic potential and a poor patient prognosis (37). miR-21 has been demonstrated to be significantly upregulated in activated PSC-derived exosomes (10). In the present study, WGCNA results revealed that high miR-21 expression was associated with a poor prognosis in patients with PAAD. KEGG analysis further showed that miR-21 primarily regulates the Ras and ERK signaling pathways and activates Ras signaling in patients with PAAD. Therefore, miR-21 may represent a key factor in pancreatic cancer onset and development. Accordingly, it was hypothesized in the present study that PSC-derived exosomal miR-21 may contribute to the malignant phenotype of pancreatic cancer cells.

In vitro experiments provided partial support for this hypothesis. Inhibition of miR-21 expression in PSC-derived exosomes resulted in decreased PDAC cell motility, possibly by inhibiting EMT and MMP-2/9 activity, demonstrating that exosomal miR-21 serves an important role in promoting PDAC cell migration. In line with the bioinformatics analysis, the in vitro transfer of exosomal miR-21 from PSCs to 
A

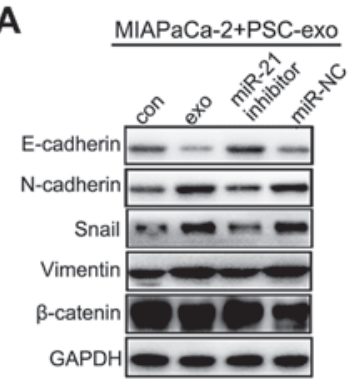

E

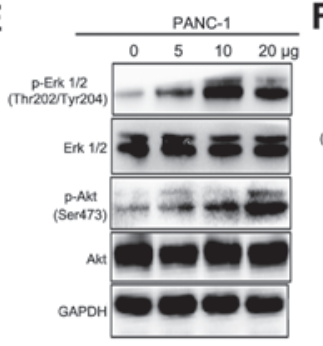

B

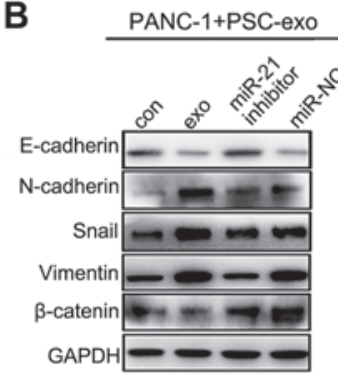

$\mathbf{F}$

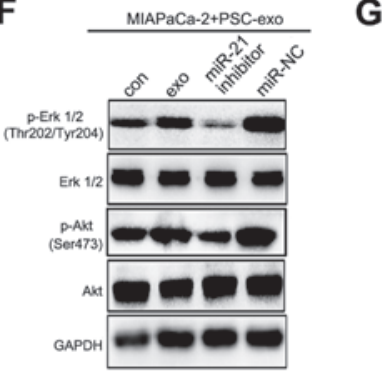

C

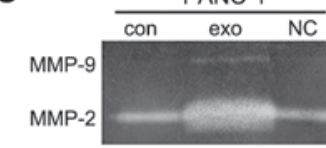

D

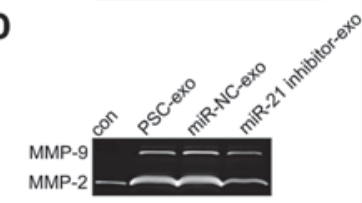

G

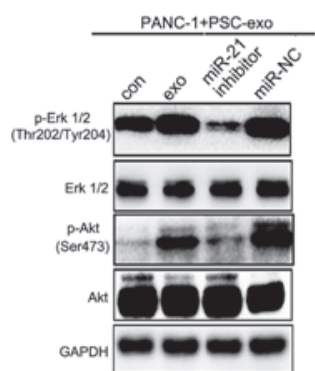

H

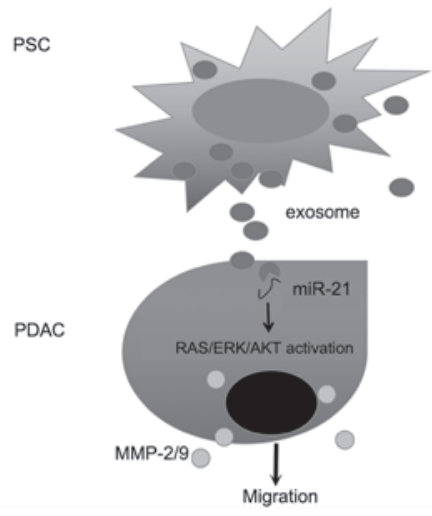

Figure 4. PSC-derived exosomal miR-21 induces EMT and increases MMP-2/9 and Ras/ERK/Akt signaling activity. (A and B) MIAPaCa-2 and PANC-1 cells were treated with exosomes from untreated PSCs or PSCs treated with the miR-21 inhibitor for $48 \mathrm{~h}$. Levels of EMT-related proteins were analyzed by western blotting. (C) MMP-2/9 activity in PANC-1 cells treated with PSC-derived exosomes. (D) MMP-2/9 activity in MIAPaCa-2 and PANC-1 cells treated with exosomes from PSCs transfected with an miR-21 inhibitor. (E) The effect of treatment with PSC-derived exosomes $(0,5,10$ or $20 \mu \mathrm{g})$ on phospho-Akt and phospho-ERK levels in PANC-1 cells. (F and G) Phospho-Akt and phospho-ERK levels in MIAPaCa-2 and PANC-1 cells following treatment with exosomes obtained from PSCs treated with the miR-21 inhibitor. (H) Summary figure of the interaction model and signaling pathways between PSCs and PDAC. PSC, pancreatic stellate cell; miR, microRNA; EMT, epithelial-mesenchymal transition; MMP, matrix metalloproteinase; p-, phospho-; NC, negative control cells treated with the supernatant from exosome cultures; exo, exosome-enriched conditioned medium; con, untreated control cells; PDAC, pancreatic ductal adenocarcinoma.

neighboring cancer cells led to increased activation of the Ras/ERK and Ras/Akt signaling pathways and expression of the EMT-associated genes and MMP-2/9. By contrast, inhibition of exosomal miR-21 decreased the activation of Ras signaling. These results suggests that PSC-derived exosomes induced EMT and increased the migratory capacity of PDAC cells via the activation of Ras/ERK and Ras/Akt signaling. Although previous studies have reported that miR-21 regulates Ras/ERK and Ras/Akt signaling by repressing Ras P21 protein activator 1 translation $(38,39)$, to the best of our knowledge, the present study is the first to demonstrate that PSC-derived exosomal miR-21 transfer promotes the activation of these two signaling pathways in PDAC cells.

This study had some limitations. In addition to miRNAs, exosomes also contain mRNAs, DNA fragments and various proteins (5); therefore, the effects of exosomes on the malignant phenotype observed in PDAC cells may be more complex and variable than the sole influence of miR-21. Thus, further studies should assess other potential effects such as cell proliferation, apoptosis and drug resistance (40). In addition, the roles of other miRNAs differentially expressed between cancer and healthy tissues should be explored. Additionally, exosomal miR-21 expression levels need to be analyzed in a larger panel of PSCs and PDAC cells to validate the results of the present study. Finally, the effects of exosomal miR-21 on PDAC metastasis should be further analyzed in vivo.

In conclusion, the results of the present study suggested that PSC-derived exosomes were released into the tumor microenvironment to promote PDAC EMT and migration via miR-21-induced activation of the Ras/ERK and Ras/Akt signaling pathways. These results highlighted PSC-derived exosomal miR-21 as a novel molecular determinant of PSC-PDAC cell communication to promote EMT induction, PDAC metastasis and progression, suggesting a potential new target for treatment or prognosis prediction for patients with pancreatic cancer. 


\section{Acknowledgements}

Not applicable.

\section{Funding}

This work was supported by the National Natural Science Foundation of China (grant nos. 81502625, 81602162 and 31471366), the National Key Research and Development Program of China (grant no. 2016YFC0901500) and the Center for Molecular Pathology, Chinese Academy of Medicine Science and Peking Union Medical College, Beijing, China (grant no. 2017PT31008).

\section{Availability of data and materials}

The datasets used and/or analyzed during the current study are available from the corresponding author on reasonable request.

\section{Authors' contributions}

HWW, ZYL and THL conceived and designed the study. QM and YX performed the experiments and data analysis. QM and ZYL wrote, reviewed and revised the manuscript. HWW and YX provided technical support.

\section{Ethics approval and consent to participate}

This study was approved by the Research Ethics Boards of Peking Union Medical College Hospital. All patients provided written informed consent prior to the study.

\section{Patient consent for publication}

Not applicable.

\section{Competing interests}

The authors declare that they have no competing interests.

\section{References}

1. Subramani R, Lopez-Valdez R, Arumugam A, Nandy S, Boopalan $\mathrm{T}$ and Lakshmanaswamy R: Targeting insulin-like growth factor 1 receptor inhibits pancreatic cancer growth and metastasis. PLoS One 9: e97016, 2014.

2. McGuigan A, Kelly P, Turkington RC, Jones C, Coleman HG and McCain RS: Pancreatic cancer: A review of clinical diagnosis, epidemiology, treatment and outcomes. World J Gastroenterol 24 4846-4861, 2018.

3. Razidlo GL, Magnine C, Sletten AC, Hurley RM, Almada LL, Fernandez-Zapico ME, Ji B and McNiven MA: Targeting Pancreatic Cancer Metastasis by Inhibition of Vav1, a Driver of Tumor Cell Invasion. Cancer Res 75: 2907-2915, 2015.

4. Wang $\mathrm{H}$ and Chen L: Tumor microenviroment and hepatocellular carcinoma metastasis. J Gastroenterol Hepatol 28 (Suppl 1): $43-48,2013$

5. Kahlert $C$ and Kalluri R: Exosomes in tumor microenvironment influence cancer progression and metastasis. J Mol Med (Berl) 91: 431-437, 2013.

6. Gajos-Michniewicz A, Duechler M and Czyz M: MiRNA in melanoma-derived exosomes. Cancer Lett 347: 29-37, 2014

7. Li L, Li C, Wang S, Wang Z, Jiang J, Wang W, Li X, Chen J, Liu K, Li C, et al: Exosomes Derived from Hypoxic Oral Squamous Cell Carcinoma Cells Deliver miR-21 to Normoxic Cells to Elicit a Prometastatic Phenotype. Cancer Res 76: 1770-1780, 2016.
8. Liao J, Liu R, Shi YJ, Yin LH and Pu YP: Exosome-shuttling microRNA-21 promotes cell migration and invasion-targeting PDCD4 in esophageal cancer. Int J Oncol 48: 2567-2579, 2016.

9. Takikawa T, Masamune A, Yoshida N, Hamada S, Kogure T and Shimosegawa T: Exosomes Derived From Pancreatic Stellate Cells: MicroRNA Signature and Effects on Pancreatic Cancer Cells. Pancreas 46: 19-27, 2017.

10. Ali S, Suresh R, Banerjee S, Bao B, Xu Z, Wilson J, Philip PA, Apte $\mathrm{M}$ and Sarkar FH: Contribution of microRNAs in understanding the pancreatic tumor microenvironment involving cancer associated stellate and fibroblast cells. Am J Cancer Res 5: 1251-1264, 2015.

11. Charrier A, Chen R, Chen L, Kemper S, Hattori T, Takigawa M and Brigstock DR: Connective tissue growth factor (CCN2) and microRNA-21 are components of a positive feedback loop in pancreatic stellate cells (PSC) during chronic pancreatitis and are exported in PSC-derived exosomes. J Cell Commun Signal 8: 147-156, 2014.

12. Bachem MG, Schneider E, Gross H, Weidenbach H, Schmid RM, Menke A, Siech M, Beger H, Grünert A and Adler G: Identification, culture, and characterization of pancreatic stellate cells in rats and humans. Gastroenterology 115: 421-432, 1998.

13. Au Yeung CL, Co NN, Tsuruga T, Yeung TL, Kwan SY, Leung CS, Li Y, Lu ES, Kwan K, Wong KK, et al: Exosomal transfer of stroma-derived miR21 confers paclitaxel resistance in ovarian cancer cells through targeting APAF1. Nat Commun 7: 11150, 2016.

14. Chandrashekar DS, Bashel B, Balasubramanya SAH, Creighton CJ, Ponce-Rodriguez I, Chakravarthi BVSK and Varambally S: UALCAN: A portal for facilitating tumor subgroup gene expression and survival analyses. Neoplasia 19: 649-658, 2017.

15. Langfelder $P$ and Horvath S: WGCNA. an R package for weighted correlation network analysis. BMC Bioinformatics 9: 559, 2008.

16. Xu Y, Li H, Huang C, Zhao T, Zhang H, Zheng C, Ren H and Hao J: Wnt2 protein plays a role in the progression of pancreatic cancer promoted by pancreatic stellate cells. Med Oncol 32: 97, 2015.

17. Wrighton KH: Cell migration: EMT promotes contact inhibition of locomotion. Nat Rev Mol Cell Biol 16: 518, 2015.

18. El-Ghlban S, Kasai T, Shigehiro T, Yin HX, Sekhar S, Ida M, Sanchez A, Mizutani A, Kudoh T, Murakami H, et al: Chlorotoxin-Fc fusion inhibits release of MMP-2 from pancreatic cancer cells. BioMed Res Int 2014: 152659, 2014.

19. Xu LF, Wu ZP, Chen Y, Zhu QS, Hamidi S and Navab R: MicroRNA-21 (miR-21) regulates cellular proliferation, invasion, migration, and apoptosis by targeting PTEN, RECK and Bcl-2 in lung squamous carcinoma, Gejiu City, China. PLoS One 9: e103698, 2014.

20. Jiao Y, Fu Z, Li Y, Meng L and Liu Y: High EIF2B5 mRNA expression and its prognostic significance in liver cancer: A study based on the TCGA and GEO database. Cancer Manag Res 10: 6003-6014, 2018.

21. Cerami E, Gao J, Dogrusoz U, Gross BE, Sumer SO, Aksoy BA, Jacobsen A, Byrne CJ, Heuer ML, Larsson E, et al: The cBio cancer genomics portal: An open platform for exploring multidimensional cancer genomics data. Cancer Discov 2: 401-404, 2012.

22. Szklarczyk D, Morris JH, Cook H, Kuhn M, Wyder S, Simonovic M, Santos A, Doncheva NT, Roth A, Bork P, et al: The STRING database in 2017: Quality-controlled protein-protein association networks, made broadly accessible. Nucleic Acids Res 45 (D1): D362-D368, 2017.

23. Livak KJ and Schmittgen TD: AAnalysis of relative gene expression data using real-time quantitative PCR and the 2- $\Delta \Delta \mathrm{CT}$ method. Methods 25: 402-408, 2001.

24. Tiwari P, Sahay S, Pandey M, Qadri SS and Gupta KP: Preventive effects of butyric acid, nicotinamide, calcium glucarate alone or in combination during the 7,12-dimethylbenz (a) anthracene induced mouse skin tumorigenesis via modulation of K-Ras-PI3K-AKTpathway and associated micro RNAs. Biochimie 121: 112-122, 2016.

25. Nussinov R, Muratcioglu S, Tsai CJ, Jang H, Gursoy A and Keskin O: The Key Role of Calmodulin in KRAS-Driven Adenocarcinomas. Mol Cancer Res 13: 1265-1273, 2015.

26. Vena F, Li Causi E, Rodriguez-Justo M, Goodstal S, Hagemann T, Hartley JA and Hochhauser D: The MEK1/2 Inhibitor Pimasertib Enhances Gemcitabine Efficacy in Pancreatic Cancer Models by Altering Ribonucleotide Reductase Subunit-1 (RRM1). Clin Cancer Res 21: 5563-5577, 2015. 
27. Sahu N, Chan E, Chu F, Pham T, Koeppen H, Forrest W, Merchant $M$ and Settleman J: Co-targeting of MEK and PDGFR/STAT3 pathways to treat pancreaticductal adenocarcinoma. Mol Cancer Ther 16: 1729-1738, 2017.

28. Xu Z, Vonlaufen A, Phillips PA, Fiala-Beer E, Zhang X, Yang L, Biankin AV, Goldstein D, Pirola RC, Wilson JS, et al: Role of pancreatic stellate cells in pancreatic cancer metastasis. Am J Pathol 177: 2585-2596, 2010.

29. Xu Z, Pothula SP, Wilson JS and Apte MV: Pancreatic cancer and its stroma: A conspiracy theory. World J Gastroenterol 20: 11216-11229, 2014.

30. Masamune A and Shimosegawa T: Pancreatic stellate cells: A dynamic player of the intercellular communication in pancreatic cancer. Clin Res Hepatol Gastroenterol 39 (Suppl 1): S98-S103, 2015.

31. Prakash J: Cancer-Associated Fibroblasts: Perspectives in Cancer Therapy. Trends Cancer 2: 277-279, 2016.

32. Hu Y, Yan C, Mu L, Huang K, Li X, Tao D, Wu Y and Qin J: Fibroblast-Derived Exosomes Contribute to Chemoresistance through Priming Cancer Stem Cells in Colorectal Cancer. PLoS One 10: e0125625, 2015.

33. Donnarumma E, Fiore D, Nappa M, Roscigno G, Adamo A, Iaboni M, Russo V, Affinito A, Puoti I, Quintavalle C, et al: Cancer-associated fibroblasts release exosomal microRNAs that dictate an aggressive phenotype in breast cancer. Oncotarget 8: 19592-19608, 2017.
34. Forterre A, Jalabert A, Chikh K, Pesenti S, Euthine V, Granjon A, Errazuriz E, Lefai E, Vidal H and Rome S: Myotube-derived exosomal miRNAs downregulate Sirtuin1 in myoblasts during muscle cell differentiation. Cell Cycle 13: 78-89, 2014

35. Gao W, Xu J, Liu L, Shen H, Zeng H and Shu Y: A systematicanalysis of predicted miR-21 targets identifies a signature for lung cancer. Biomed Pharmacother 66: 21-28, 2012.

36. Di Leva G, Garofalo M and Croce CM: MicroRNAs in cancer. Annu Rev Pathol 9: 287-314, 2014.

37. Pfeffer SR, Yang CH and Pfeffer LM: The Role of miR-21 in Cancer. Drug Dev Res 76: 270-277, 2015.

38. Sharma SB, Lin CC, Farrugia MK, McLaughlin SL, Ellis EJ, Brundage KM, Salkeni MA and Ruppert JM: MicroRNAs 206 and 21 cooperate to promote RAS-extracellular signal-regulated kinase signaling by suppressing the translation of RASA 1 and SPRED1. Mol Cell Biol 34: 4143-4164, 2014.

39. Liu H, Huang X, Liu X, Xiao S, Zhang Y, Xiang T, Shen X, Wang $G$ and Sheng B: miR-21 promotes human nucleus pulposus cell proliferation through PTEN/AKT signaling. Int J Mol Sci 15: 4007-4018, 2014.

40. Zhang L, Yao J, Li W and Zhang C: Micro-RNA-21 Regulates Cancer-Associated Fibroblast-Mediated Drug Resistance in Pancreatic Cancer. Oncol Res 26: 827-835, 2018. 